\title{
An Energy-Based State Observer for Dynamical Subsystems with Inaccessible State Variables
}

\author{
Islam S. M. Khali1*, Asif Sabanovic ${ }^{\dagger}$ and Sarthak Misra*
}

\begin{abstract}
This work presents an energy-based state estimation formalism for a class of dynamical systems with inaccessible/unknown outputs, and systems at which sensor utilization is impractical, or when measurements can not be taken. The power-conserving physical interconnections among most of the dynamical subsystems allow for power exchange through their power ports. Power exchange is conceptually considered as information exchange among the dynamical subsystems and further utilized to develop a natural feedback-like information from a class of dynamical systems with inaccessible/unknown outputs. This information is used in the design of an energybased state observer. Convergence stability of the estimation error for the proposed state observer is proved for systems with linear dynamics. Furthermore, robustness of the convergence stability is analyzed over a range of parameter deviation and model uncertainties. Experiments are conducted on a dynamical system with a single input and multiple inaccessible outputs (Fig. 1) to demonstrate the validity of the proposed energybased state estimation formalism.
\end{abstract}

\section{INTRODUCTION}

Recent research efforts in the design of mechatronics systems have been partially devoted to the problem of how to have sensors embedded to these systems, and how to overcome their associated problems. Mechanically, mechatronics systems have to be designed and manufactured such that several sensors criterions are met such as accuracy, alignment and including enough space for sensors with their associated electronics setups and complex wirings (from a control viewpoint on the other hand, measurement noise, uncertainties, hysteresis and non-collocation problems). Therefore, it would be natural to devise state observers to estimate the state variables of a dynamical system. However, the current state observers require taking measurements to be used as a basis of the estimation process that in turn necessitates having at least few sensors embedded to these systems. The sensors-associated problems limit the usefulness of many state variables estimation and control frameworks due to several aspects including, but not limited to, their noisy outputs, their limited bandwidth due to their physical structures and the complexity they add to the control systems.

The high-gain observer presented by Khalil et al. [1] shows excellent robustness properties for large enough observer gains. The practical difficulty is, however, the determination of proper observer gain due to the trade-off

*Islam S. M. Khalil and Sarthak Misra are with MIRA-Institute for Biomedical Technology and Technical Medicine, University of Twente, 7500 AE Enschede, The Netherlands \{i.s.m.khalil, s.misra\} @utwente.nl

${ }^{\dagger}$ Asif Sabanovic is with the Faculty of Engineering and Natural Sciences, Sabanci University, 34956, Turkey asifesabanciuniv.edu

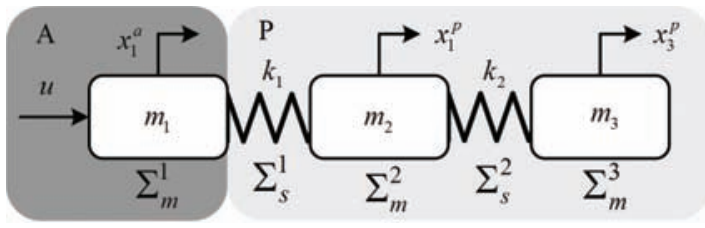

Fig. 1. Dynamical system with single input $(u)$ consisting of subsystems $\mathbf{A}$ and $\mathbf{P}$. Subsystem $\mathbf{P}$ has state variables which are inaccessible for measurement, whereas measurements can be taken from subsystem $\mathbf{A}$. Effort-force along the power-conserving interconnection of subsystems $\mathbf{A}$ and $\mathbf{P}$ is considered as a natural feedback and utilized in the design of an energy-based state observer to estimate the states of subsystem $\mathbf{P}$ from the available states of subsystem $\mathbf{A}$. The mass and spring energy storage elements are labeled with $\sum_{m}$ and $\sum_{s}$, respectively. Positions of the first, second and third degrees-of-freedom are $x_{1}^{a}, x_{1}^{p}$ and $x_{3}^{p}$, respectively. The mass energy storage elements are $m_{1}, m_{2}$ and $m_{3}$, whereas the spring energy storage elements are $k_{1}$ and $k_{2}$.

between the desired bounds on the observer error and the sensitivity to noise. An adaptation scheme was presented in [2], for adjustment of the high-gain observer gain such that its advantages are retained. The trade-off between speed of state reconstruction and immunity to measurements noise was studied in [3], and a method was proposed by switching the high-gain matrix between two values, high gain during the transient to quickly recover the state reconstruction, then once a steady state error threshold has reached, the observer gain is switched to another gain to reduce the effect of measurement noise.

The well-known Luenberger observer provides a comprehensive solution for the estimation problem, where system states can be observed along with the disturbances which can be considered as state variables provided that the model of the dynamical system is known a priori, inputs are known and outputs are accessible [4]. The Luenberger observer is a very useful tool for estimating the internal variables of the system, the main challenge is, however, the complete dependence on the accuracy of the mathematical model. Based on the sliding mode approach, robustness over a range of system uncertainties was enhanced by the sliding mode observer presented by Utkin et al. [5]. A key feature in their observer is the introduction of the well-known switching function to achieve a sliding mode and stable error dynamics. This switching function was claimed to result in an excellent system performance, i.e., disturbance rejection and insensitivity to parameter deviation. In [6], a sliding mode functional observer was introduced, including the same switching function to inherit the robustness and insensitivity of the conventional sliding mode observer. The sliding mode 
functional observer, in addition, has a lower order that is the characteristic of functional observers. Authors in [7], utilized sliding mode observer to estimate derivative of measured signals in the presence of unmatched disturbances by filtering discontinuities approximations of the derivatives. Trajectory tracking controller for robot with flexible joints was presented in [8], based on feedback linearization and extended state observer, showing robustness in the presence of model uncertainties. The concept of functional observability and detectability were introduced in [9], that ascertains the ability to estimate a given linear function of the state vector using dynamical observer.

Without any loss of generality, Mariaana et al. [10], pointed out that there exist at least two major problems that makes it difficult to automate micromanipulation systems, namely, the poor understanding of the interaction phenomena and the difficulty of making measurements at micro scale. Therefore, the aforementioned state observers are hard to be implemented for such applications since measurements can not be made. Many attempts have been conducted to embed sensors with sophisticated mechatronics systems due to the dependence of the state observers and control systems on certain measurements [11], [12].

This work is concerned with exploring alternatives (natural feedback [13], [14]) for the inaccessible state variables for measurements, or alternatives for the measurements that add many drawbacks to the control system (limited band width, noise, non-collocation, complexity, etc). The power flow along the interconnected physical systems is conceptually considered as a natural feedback from a dynamical subsystem with inaccessible state variables or outputs. It was shown in [15]-[17] that the interconnected subsystems are in feedback, i.e, interactions of the dynamical subsystems result in an exchange of power through the power ports of the system.

The remainder of this paper is organized as follows: In Section II, the concept of natural feedback [13] is introduced based on the energy-based formalism and the implicit port Hamiltonian representation. In Section III, the natural feedback is utilized in designing energy-based state observer. In addition, stability margins (gain and phase margins) of the energy-based state observer are analyzed. Experimental results are provided in Section IV. Finally, Section V concludes and provides final remarks and directions for future work.

\section{NATURAL FEEDBACK}

Considering an $n$-dimensional dynamical system which consists of subsystem/plant $(\mathbf{P})$ and subsystem/actuator $(\mathbf{A})$, both subsystems are interacting through a power-conserving interconnection. Subsystem $\mathbf{P}$ has $(n-r)$ state variables $\left(\mathbf{x}^{\mathbf{P}} \in \mathbb{R}^{n-r}\right)$ that are not available for measurement, whereas the remaining $(r)$ state variables $\left(\mathbf{x}^{\mathbf{A}} \in \mathbb{R}^{r}\right)$ of subsystem (A), are available for measurement. Further, $x_{i}^{p}$ and $x_{l}^{a}$ are the $i$ th and $l$ th state variables of subsystem $\mathbf{P}$ and A, respectively. where $i=1, \ldots, n-r$, and $l=1, \ldots, r$. Dynamics of this system is illustrated in Fig. 1. Although

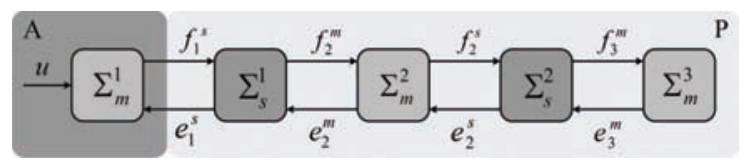

Fig. 2. Flow and effort pairs along the power ports of the dynamical system shown in Fig. 1. This representation indicates the power (information) flow along the dynamical system. The information flow along the dynamical system shows that even in the absence of the state variables of subsystem $\mathbf{P}$, there exist a natural feedback in terms of flow or effort variables which can be determined from the available states of the subsystem A. Subsystem A has a single input $u$. The mass and spring energy storage elements are labeled with $\sum_{m}$ and $\sum_{s}$, respectively. The flow and effort pair at the $i$ th power port are $f_{i}$ and $e_{i}$, respectively. The superscripts $m$ and $s$ indicates the mass and the spring energy storage elements, respectively.

the state variables of the subsystem $\mathbf{P}$ are conceptually considered inaccessible, the power exchange along the energy port between subsystems $\mathbf{A}$ and $\mathbf{P}$ indicates the flow of information from subsystem $\mathbf{P}$ to subsystem $\mathbf{A}$ and vise versa, in terms of flow or effort variables as shown in Fig. 2. In order to clarify the energy exchange along the energy ports between the interconnected subsystems, we represent the system in its port Hamiltonian form to determine the interconnection matrix $(\mathbf{J}(\mathbf{x}))$, the Hamiltonian energy function $(H(\mathbf{x}, \mathbf{p}))$ is given by

$$
H(\mathbf{x}, \mathbf{p})=\frac{p_{1}^{2}}{2 m_{1}}+\frac{p_{2}^{2}}{2 m_{2}}+\frac{p_{3}^{2}}{2 m_{3}}+\frac{k_{1}}{2} \Delta x_{21}^{2}+\frac{k_{2}}{2} \Delta x_{32}^{2},
$$

where $\mathbf{p}$ is the momentum vector. Further, $p_{k}$ and $m_{k}$ are the momentum and mass of the $k$ th mass energy storage element $(k=1, \ldots, n=3)$, and $k_{1}$ and $k_{2}$ are the stiffness of the first and second spring energy storage element, respectively. Furthermore, $\Delta x_{21}=x_{1}^{a}-x_{1}^{p}$ and $\Delta x_{32}=x_{2}^{p}-x_{1}^{p}$. The following port Hamiltonian representation encodes the interconnections between the various elements of the dynamical system:

$$
\mathbf{x}=\underbrace{\left[\begin{array}{cccccc}
0 & 1 & 0 & 0 & 0 & 0 \\
-1 & 0 & 0 & 0 & 0 & 0 \\
0 & 0 & 0 & 1 & 0 & 0 \\
0 & 0 & -1 & 0 & 1 & 0 \\
0 & 0 & 0 & -1 & 0 & 1 \\
0 & 0 & 0 & 0 & -1 & 0
\end{array}\right]}_{\mathbf{J}(\mathbf{x})}\left[\begin{array}{c}
\frac{\partial H}{\partial x_{1}} \\
\frac{\partial H}{\partial p_{1}} \\
\frac{\partial H}{\partial x_{2}} \\
\frac{\partial H}{\partial p_{2}} \\
\frac{\partial H}{\partial x_{3}} \\
\frac{\partial H}{\partial p_{3}}
\end{array}\right]+\left[\begin{array}{l}
0 \\
1 \\
0 \\
0 \\
0 \\
0
\end{array}\right] u, \quad(2)
$$

where $\mathrm{x} \in \mathbb{R}^{n}$ is the state variables of the system which consists of subsystems $\mathbf{A}$ and $\mathbf{P}$. Further, $u$ is a scalar input to subsystem A. It can be shown from (2) that, through the power-conserving power port between the dynamical subsystems $\mathbf{A}$ and $\mathbf{P}$, energy exchange occurs in terms of effort and flow variables. In the current system, the effort is the generalized force, whereas the flow is the generalized velocity.

The first mass of subsystem $\mathbf{A}$ acts on the first spring energy storage element of the subsystem $\mathbf{P}$ with a generalized velocity, the spring by its turn integrates this velocity to determine its own deflection. A generalized force 


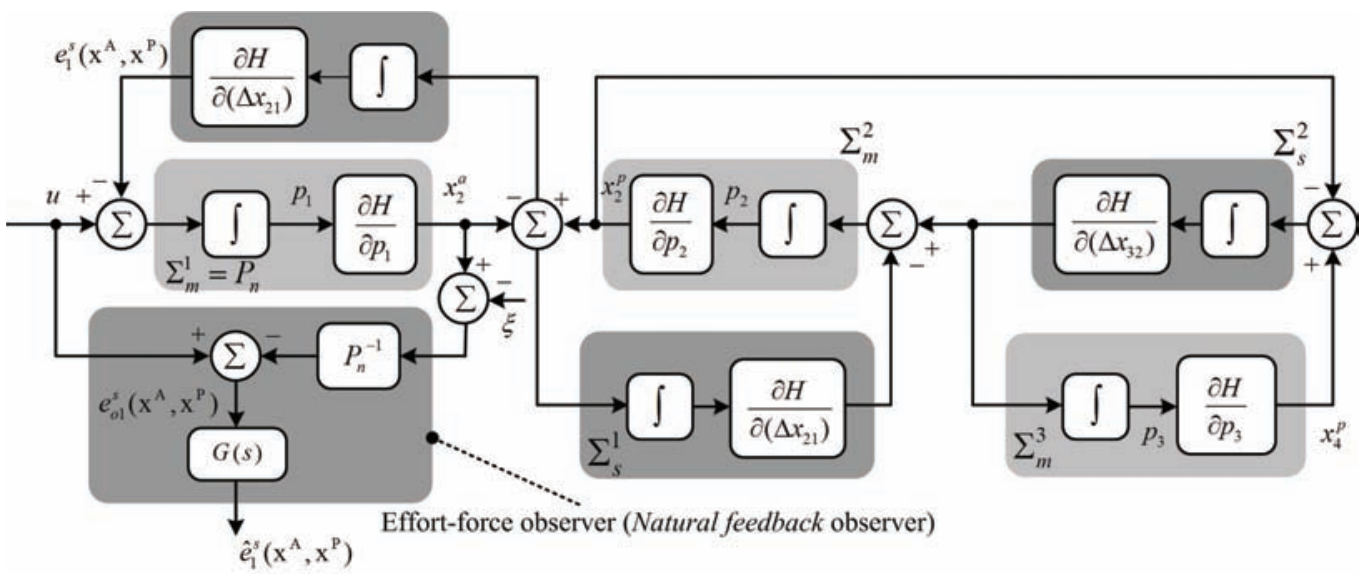

Fig. 3. Effort-force observer estimating the incident effort-force from the power port between the dynamical subsystems $\mathbf{A}$ and $\mathbf{P}$. The dynamical subsystem $\mathbf{P}$ does not have any accessible state variables for measurement, the effort-force $\left(e_{1}^{s}\left(\mathbf{x}^{\mathbf{A}}, \mathbf{x}^{\mathbf{P}}\right)\right)$ is therefore considered as a natural feedback that can be determined by the effort-force observer based on measurements taken from subsystem $\mathbf{A}$. The estimated natural feedback effort-force $\left(\widehat{e}_{1}^{s}\left(u, \mathbf{x}^{\mathbf{A}}\right)\right)$ is injected onto the observer structure (9) to enforce stable estimation error dynamics due to the absence of the state variables of subsystem $\mathbf{P}$. Subsystem $\mathbf{A}$ has a single input $(u)$. The mass and spring energy storage elements are labeled with $\sum_{m}$ and $\sum_{s}$, respectively. Further, $H(\mathbf{x}, \mathbf{p})$ is the Hamiltonian energy function and $\xi$ is the measurement noise. The effort-force observer depends on the applied input $(u)$ and a single measurement from subsystem A, i.e., velocity of the first degree-of-freedom $\left(x_{2}^{a}\right)$.

is generated by taking the partial derivative of the spring potential energy with respect to the deflection, this effortforce (natural feedback) is the output of the dynamical subsystem $\mathbf{P}$ which acts as an input on subsystem $\mathbf{A}$. The mass energy storage element integrates this input force to determine its own momentum. Its velocity is then determined by taking the partial derivative of its kinetic energy with respect to its momentum. The interconnection between the two dynamical subsystems is in feedback. Therefore, there exist a natural feedback in terms of effort or flow variables from the dynamical subsystem $\mathbf{P}$ on $\mathbf{A}$. The system we consider is fairly general since energy storage and dissipation elements along with gyrators can be used to model any complex linear or nonlinear systems (only energy storage elements are analyzed in this work).

It will now be shown that this effort-force can be estimated through the available variables of subsystem A. In our dynamical system, the natural feedback is the effort-force $\left(e_{1}^{s}\left(\mathbf{x}^{\mathbf{A}}, \mathbf{x}^{\mathbf{P}}\right)\right)$. Since this natural feedback effort-force is a function of time, it can be approximated by a polynomial with a proper order $(z)[18]$

$$
\frac{d^{z}}{d t^{z}} e_{1}^{s}(t)=0
$$

The natural feedback effort-force can be approximated by a step function, when the order of the polynomial (3) is, $z=1$, and can be approximated by a ramp function, when the order of the polynomial is, $z=2$, similarly, a parabolic approximation of the natural feedback effort-force requires the order of the polynomial to be, $z=3$, and so forth. Using the available variables of subsystem $\mathbf{A}$ along with its nominal model, the calculated natural feedback effort-force is given by

$$
\begin{aligned}
e_{o 1}^{s}\left(u, \mathbf{x}^{\mathbf{A}}\right) & =u-P_{n}^{-1}\left(x_{2}^{a}-\xi\right) \\
& =\left(P^{-1}-P_{n}^{-1}\right) x_{2}^{a}-e_{1}^{s}\left(\mathbf{x}^{\mathbf{A}}, \mathbf{x}^{\mathbf{P}}\right)+P_{n}^{-1} \xi
\end{aligned}
$$

where, $e_{o 1}^{s}\left(u, \mathbf{x}^{\mathbf{A}}\right)$ and $e_{1}^{s}\left(\mathbf{x}^{\mathbf{A}}, \mathbf{x}^{\mathbf{P}}\right)$ are the calculated natural feedback effort-force through the nominal dynamics of subsystem $\mathbf{A}$, and the actual natural feedback effortforce, respectively. Further, $P$ and $P_{n}$ are the actual and nominal models of subsystem $\mathbf{A}$ or $\sum_{m}^{1}$. Furthermore, $\xi$ is the measurement input noise. Due to presence of differentiator in the inverse dynamics of subsystem $\mathbf{A}$, the natural feedback effort-force has to be calculated through a low-pass filter $(G(s))$

$$
\widehat{e}_{1}^{s}\left(u, \mathbf{x}^{\mathbf{A}}\right)=G(s) e_{o 1}^{s}\left(u, \mathbf{x}^{\mathbf{A}}\right)=\frac{g_{e f f}}{s+g_{e f f}} e_{o 1}^{s}\left(u, \mathbf{x}^{\mathbf{A}}\right),
$$

where $\widehat{e}_{1}^{s}\left(u, \mathbf{x}^{\mathbf{A}}\right)$ is the estimated natural feedback effortforce. Further, $g_{\text {eff }}$ is the cut-off frequency of the low-pass filter $(G(s))$ with first order for simplicity. The low-pass filter $(G(s))$ has a bandwidth that is limited by the bandwidth of the measurement noise, and its order depends on the degree of the polynomial (3) used to model the natural feedback effort-force. The effort-force observer is depicted in Fig. 3 which indicates that even in the absence of state variables or outputs of subsystem $\mathbf{P}$, a natural feedback effort-force exists and can be calculated from the available variables $(u$ and $\mathbf{x}^{\mathbf{A}}$ ) of subsystem $\mathbf{A}$, provided that the interconnection of these subsystems is power-conserving.

\section{ENERGY-BASED STATE OBSERVER}

It is essential to note that the exact model of the natural feedback effort-force depends on the Dirac structure which represents the power-conserving interconnection between the 


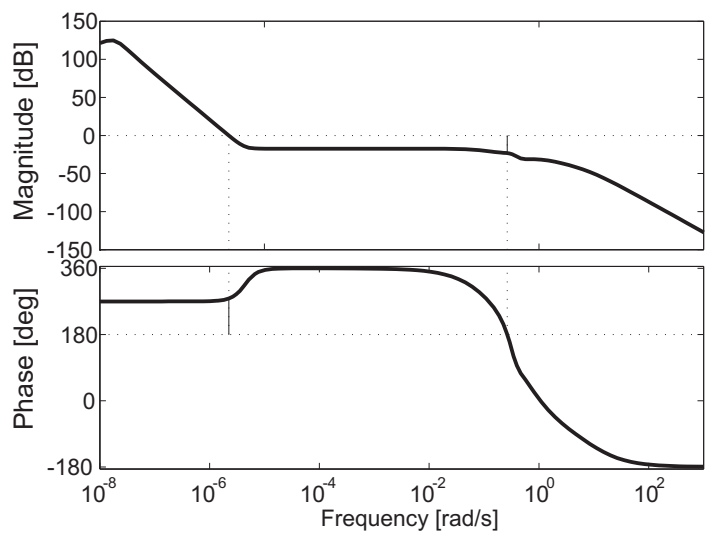

(a) $u \longmapsto \widetilde{x}_{1}^{p}$

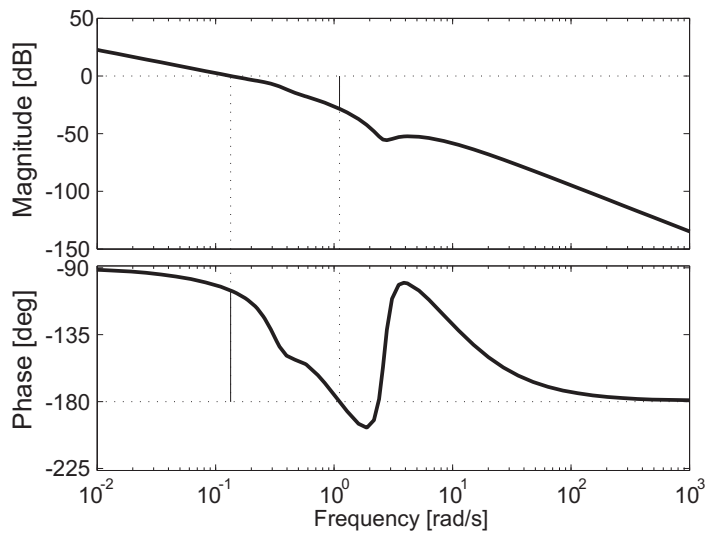

(c) $u \longmapsto \widetilde{x}_{3}^{p}$

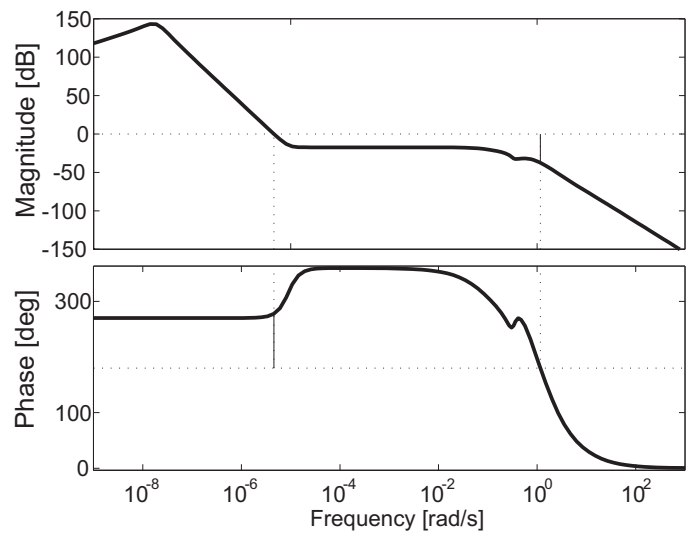

(b) $u \longmapsto \widetilde{x}_{2}^{p}$

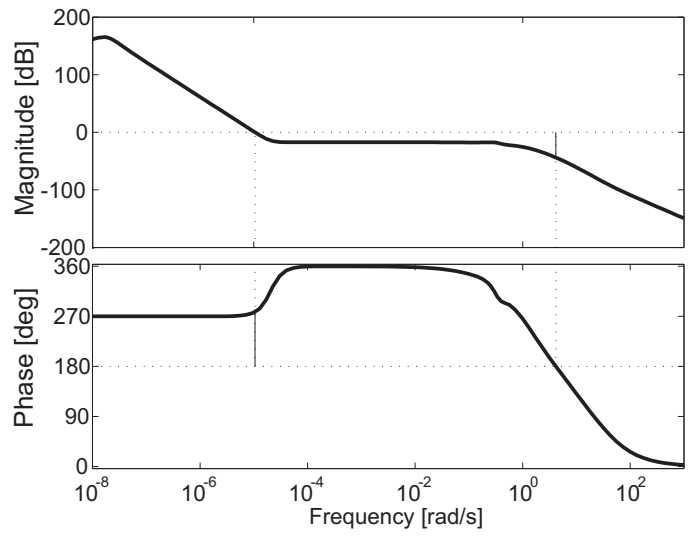

(d) $u \longmapsto \widetilde{x}_{4}^{p}$

Fig. 4. Stability margins of the energy-based state observer. The gain margin $(\mathrm{Gm})$ and phase margin $(\mathrm{Pm})$ are calculated for the derived transfer functions between single input $(u)$ and the state estimation errors $\widetilde{x}_{1}^{p}, \widetilde{x}_{2}^{p}, \widetilde{x}_{3}^{p}$ and $\widetilde{x}_{4}^{p}$, respectively. The stability margins are: (a) $\mathrm{Gm}=23.2 \mathrm{~dB}$ and Pm=97.8 deg. (b) $\mathrm{Gm}=37.5 \mathrm{~dB}$ and $\mathrm{Pm}=97.8 \mathrm{deg}$. (c) $\mathrm{Gm}=28.4 \mathrm{~dB}$ and $\mathrm{Pm}=74.8 \mathrm{deg}$. (d) $\mathrm{Gm}=44.0 \mathrm{~dB}$ and $\mathrm{Pm}=97.8 \mathrm{deg}$. The calculated gain and phase margins indicate that the energy-based state observer has enough stability margins to tolerate with the parameter deviation and phase lag.

interacting subsystems. This is the central idea in designing energy-based state observer to estimate the inaccessible state variables of the subsystem P. System (2) has the following state space representation

$$
\dot{\mathbf{x}}=\mathbf{A} \mathbf{x}+\mathbf{B} u, \mathbf{y}=\mathbf{C x}+\mathbf{D} u,
$$

where $\mathbf{x}=\left[\mathbf{x}^{\mathbf{A}} \mid \mathbf{x}^{\mathbf{P}}\right]^{\mathrm{T}} \in \mathbb{R}^{n}$ and $\mathbf{y} \in \mathbb{R}^{m}$ are the state and measurement vectors, respectively. $\mathbf{A}, \mathbf{B}, \mathbf{C}$ and $\mathbf{D}$ are the system matrix, distribution vector of the input, observation column vector and feed forward matrix with the appropriate dimensions, respectively. In order to decouple the natural feedback effort-force in the previous representation, it can be shown that (6) can be represented in the following form:

$$
\dot{\mathbf{x}}=\left[\begin{array}{c:c}
\mathbf{A}^{\mathbf{A}} & \emptyset \\
- & - \\
\emptyset & \mathbf{A}^{\mathbf{P}}
\end{array}\right] \mathbf{x}+\left[\begin{array}{c}
\mathbf{B}^{\mathbf{A}} \\
- \\
\emptyset
\end{array}\right] u+\left[\begin{array}{c}
\mathbf{B}^{e f f} \\
-- \\
\mathbf{B}^{\mathbf{P}}
\end{array}\right] e_{1}^{s}\left(\mathbf{x}^{\mathbf{A}}, \mathbf{x}^{\mathbf{P}}\right)
$$

where $\mathbf{A}^{\mathbf{A}} \in \mathbb{R}^{r \times r}$ and $\mathbf{A}^{\mathbf{P}} \in \mathbb{R}^{(n-r) \times(n-r)}$ are the system matrices of the dynamical subsystems with accessible and inaccessible state variables, respectively. Further, $\mathbf{B}^{\mathbf{A}} \in$ $\mathbb{R}^{r \times 1}, \mathbf{B}^{e f f} \in \mathbb{R}^{r \times 1}$ and $\mathbf{B}^{\mathbf{P}} \in \mathbb{R}^{(n-r) \times 1}$ are the distribution vector of the input $\left(u \in \mathbb{R}^{1 \times 1}\right)$ and distribution vector of the scalar natural feedback effort-force $\left(e_{1}^{s}\left(\mathbf{x}^{\mathbf{A}}, \mathbf{x}^{\mathbf{P}}\right)\right)$ on the actuator and plant with the proper dimensions, respectively. The function $\left(e_{1}^{s}\left(\mathbf{x}^{\mathbf{A}}, \mathbf{x}^{\mathbf{P}}\right)\right)$ depends on the Dirac structure of the power-conserving interconnections which is represented in the interconnection matrix $(\mathbf{J}(\mathbf{x}))$ of (2). According to (2), the natural feedback effort-force is

$$
e_{1}^{s}\left(\mathbf{x}^{\mathbf{A}}, \mathbf{x}^{\mathbf{P}}\right)=k_{1}\left(x_{1}^{a}-x_{1}^{p}\right),
$$

Ideally, the estimated natural feedback effort-force $\left(\widehat{e}\left(u, \mathbf{x}^{\mathbf{P}}\right)\right)$ will converge to the actual natural feedback effort-force $\left(e_{1}^{s}\left(\mathbf{x}^{\mathbf{A}}, \mathbf{x}^{\mathbf{P}}\right)\right)$ in finite time by properly designing the effort-force observer (5)

$$
\widehat{e}_{1}^{s}\left(u, \mathbf{x}^{\mathbf{A}}\right) \longmapsto e_{1}^{s}\left(\mathbf{x}^{\mathbf{A}}, \mathbf{x}^{\mathbf{P}}\right) .
$$

To be more precise, the estimated natural feedback effortforce is perturbed with the estimation error of the natural feedback effort-force $\left(\Delta e_{1}^{s}\left(\mathbf{x}^{\mathbf{A}}, \mathbf{x}^{\mathbf{P}}\right)\right)$. According to the 
structure of the effort-force observer (5), the perturbation over the estimated natural feedback effort-force is, [1 $G(s)] \Delta e_{1}^{s}\left(\mathbf{x}^{\mathbf{A}}, \mathbf{x}^{\mathbf{P}}\right)$. Therefore

$$
\widehat{e}_{1}^{s}\left(u, \mathbf{x}^{\mathbf{A}}\right)=e_{1}^{s}\left(\mathbf{x}^{\mathbf{A}}, \mathbf{x}^{\mathbf{P}}\right)+[1-G(s)] \Delta e_{1}^{s}\left(\mathbf{x}^{\mathbf{A}}, \mathbf{x}^{\mathbf{P}}\right),
$$

where $G(s)$ can be interpreted as a sensitivity function to the sensor noise, while $(1-G(s))$ is a sensitivity function to the perturbation over the estimated natural feedback effort-force. From (8), the estimated natural feedback effort-force will eventually converge to the actual one. Therefore, we devise an observer of the following form:

$$
\dot{\widehat{\mathbf{x}}}=\mathbf{A} \widehat{\mathbf{x}}+\mathbf{B} u+\mathbf{M}\left[\widehat{e}_{1}^{s}\left(u, \mathbf{x}^{\mathbf{A}}\right)-e_{1}^{s}\left(\mathbf{x}^{\mathbf{A}}, \widehat{\mathbf{x}}^{\mathbf{P}}\right)\right],
$$

where $\mathbf{M} \in \mathrm{R}^{n \times 1}$ is the gain vector of the energy-based state observer (9). Further, $\widehat{e}_{1}^{s}\left(u, \mathbf{x}^{\mathbf{A}}\right)$ is the estimated natural feedback effort-force based on the available variables of subsystem $\mathbf{A}$, and $e_{1}^{s}\left(\mathbf{x}^{\mathbf{A}}, \widehat{\mathbf{x}}^{\mathbf{P}}\right)$ is the natural feedback effortforce based on the Dirac structure of the power-conserving interconnection of subsystems $\mathbf{A}$ and $\mathbf{P}$, calculated by the estimated states of subsystem $\mathbf{P}$. Based on (8), the estimated natural feedback effort-force $\left(\widehat{e}_{1}^{s}\left(u, \mathbf{x}^{\mathbf{A}}\right)\right)$ will converge to the actual natural feedback effort-force $\left(e_{1}^{s}\left(\mathbf{x}^{\mathbf{A}}, \mathbf{x}^{\mathbf{P}}\right)\right)$. Therefore, the actual force is used in (9). The estimation error $(\widetilde{\mathbf{x}}=\mathbf{x}-\widehat{\mathbf{x}})$ can be calculated by subtracting (6) and (9), this operation yields

$$
\begin{gathered}
\dot{\tilde{\mathbf{x}}}=\mathbf{A} \widetilde{\mathbf{x}}-\mathbf{M}\left[e_{1}^{s}\left(\mathbf{x}^{\mathbf{A}}, \mathbf{x}^{\mathbf{P}}\right)-e_{1}^{s}\left(\mathbf{x}^{\mathbf{A}}, \widehat{\mathbf{x}}^{\mathbf{P}}\right)\right] \\
=\mathbf{A} \widetilde{\mathbf{x}}-\mathbf{M}\left[k_{1}\left(x^{a}-x_{i}^{p}\right)-k_{1}\left(x^{a}-\widehat{x}_{i}^{p}\right)\right]=\mathbf{A} \widetilde{\mathbf{x}}-k_{1} \mathbf{M}\left[\widehat{x}_{i}^{p}-x_{i}^{p}\right],
\end{gathered}
$$

where the index $i$ refers to the degree-of-freedom at which the power-conserving interconnection occurs between subsystems $\mathbf{A}$ and $\mathbf{P}$. Therefore, the error dynamics is given by

$$
\begin{aligned}
& \dot{\widetilde{\mathbf{x}}}=\mathbf{A} \widetilde{\mathbf{x}}+k_{1} \mathbf{M} \widetilde{x}_{i}^{p}, \widetilde{x}_{i}^{p}=x_{i}^{p}-\widehat{x}_{i}^{p}
\end{aligned}
$$

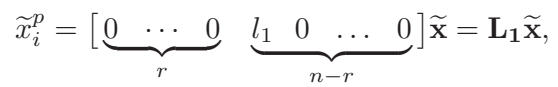

where $\mathbf{L}_{\mathbf{1}} \in \mathrm{R}^{1 \times n}, l_{1}=1$ with the index $(r+i)$ along the vector $\mathbf{L}_{1}$, this definition yields

$$
\dot{\tilde{\mathbf{x}}}=\mathbf{A} \widetilde{\mathbf{x}}+k_{1} \mathbf{M L}_{\mathbf{1}} \widetilde{\mathbf{x}}=(\mathbf{A}+\mathbf{M Z}) \widetilde{\mathbf{x}},
$$

The estimation error will vanish if the matrix $(\mathbf{A}+\mathbf{M Z}) \in$ $\mathbb{R}^{n \times n}$ is Hurwitz. The gain vector of the energy-based state observer $(\mathbf{M})$ has to be selected such that $(\mathbf{A}+\mathbf{M Z})$ is Hurwitz. This selection can be achieved by a regular pole placement procedure. If the matrix $(\mathbf{A}+\mathbf{M Z})$ is stable, the estimation error will converge to zero for any initial error vector $(\mathbf{e}(0))$, i.e., the states of subsystem $\mathbf{P}\left(\widehat{\mathbf{x}}^{\mathbf{P}}(t)\right)$ will converge to $\left(\mathbf{x}^{\mathbf{P}}(t)\right)$, regardless to the initial value of the estimated and actual states, $\widehat{\mathbf{x}}^{\mathbf{P}}(0)$ and $\mathbf{x}^{\mathbf{P}}(0)$, respectively.

The previous analysis indicates that the estimation error dynamics is not trivial, it is rather dependent on the Dirac structure representation of the interconnection between the subsystems $\mathbf{P}$ and $\mathbf{A}$. Nevertheless, the power-conserving Dirac structures are limited to few models which can be easily derived and utilized in designing similar observers and providing stable error dynamics. It is important to note that

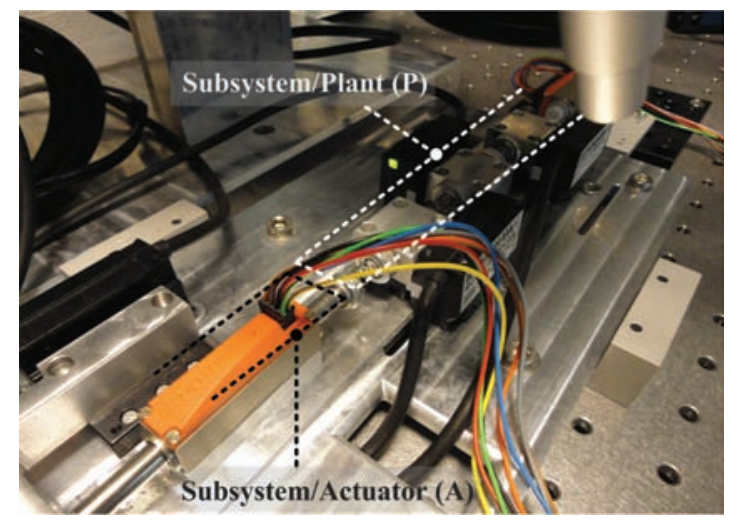

Fig. 5. Lumped mass-spring system with 4 degrees-of-freedom consisting of a single input subsystem (linear actuator representing subsystem A) attached via a spring energy storage element to a 3 degrees-of-freedom flexible system representing subsystem $\mathbf{P}$. The lumped masses of subsystem $\mathbf{P}$ are mounted on almost frictionless linear slides. Subsystem $\mathbf{P}$ has inaccessible state variables for measurement. The position encoders are used to compare the measured states to the estimated state variables by the energy-based state observer. Subsystem A is controlled to induce arbitrary excitation into subsystem $\mathbf{P}$ during the estimation of its state variables.

the design of the energy-based state observer depends on at least one scalar gain, i.e., $g_{\text {eff }}$ (first order low-pass filter is utilized in (5)), and the gain vector (M). Determination of $\left(g_{e f f}\right)$ depends on the bandwidth of the sensor noise $(\xi)$. It should be large enough to reduce the convergence time of the estimated natural feedback effort-force, on the one hand, and must be limited with the bandwidth of the sensor noise, on the other hand. Upon the desired performance of the observer, the vector gain $(\mathbf{M})$ can be determined by directly substituting the desired dominant poles $\left(\mu_{1}, \ldots, \mu_{n}\right)$ of the energy-based state observer in

$$
|s \mathbf{I}-(\mathbf{A}+\mathbf{M Z})|=\left(s-\mu_{1}\right) \ldots\left(s-\mu_{n}\right),
$$

where $\mathbf{I}$ is the identity matrix with the proper dimension.

Due to the dependence of the energy-based state observer on a single measurement from subsystem $\mathbf{A}$ along with assuming that the nominal model of subsystem $\mathbf{P}$ is known beforehand, it is important to analyze the robustness of the energy-based state observer. Transfer functions between the estimation error and the input $(u)$ for each state variable are derived to determine the stability margins of the energybased state observer as shown in Fig. 4 , where $\widetilde{x}_{i}^{p}$ represents the estimation error of the $i$ th state variable of subsystem $\mathbf{P}$. It can be shown from Fig. 4 that the stability margins are large enough which allow the energy-based state observer to tolerate with deviations over the nominal parameters of (5) and (9). In addition, the phase margins provide the system with enough stability margin to tolerate with the phase lag induced by the energy-based state observer.

\section{EXPERIMENTAL RESULTS}

In order to experimentally examine the validity of the proposed energy-based state observer, experiments are con- 


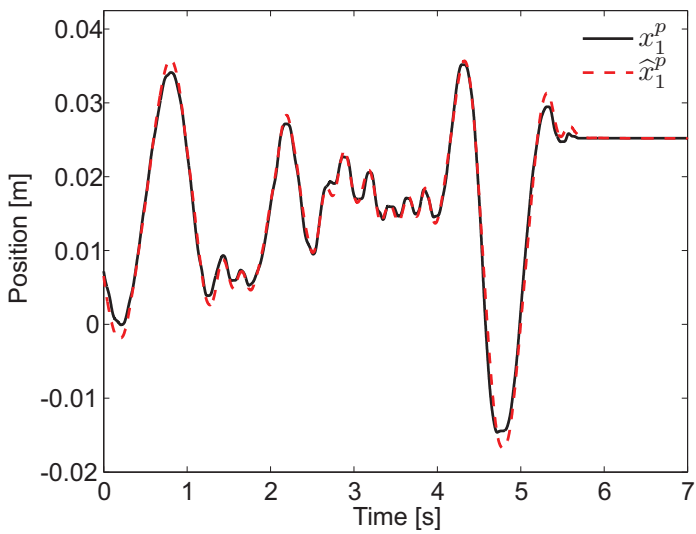

(a) Estimated versus actual position of the 1st mass of subsystem $\mathbf{P}$

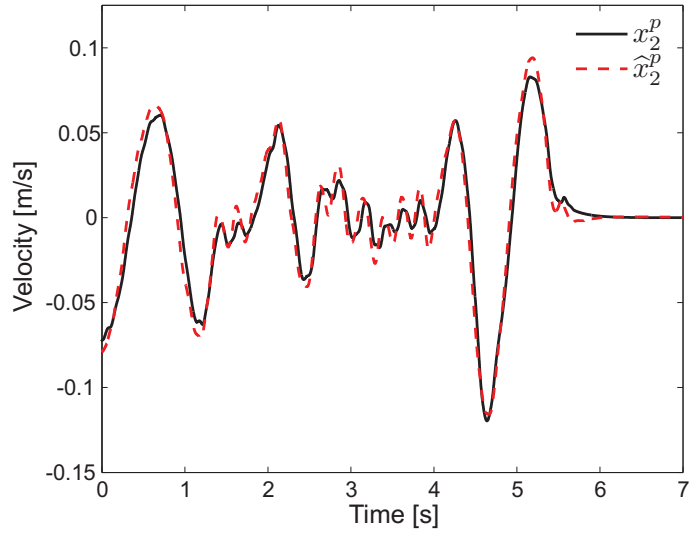

(c) Estimated versus actual velocity of the 1 st mass of subsystem $\mathbf{P}$

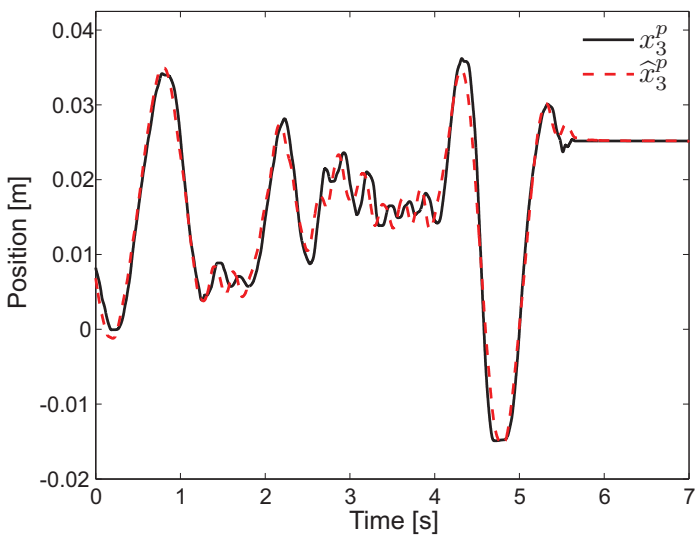

(b) Estimated versus actual position of the 2nd mass of subsystem $\mathbf{P}$

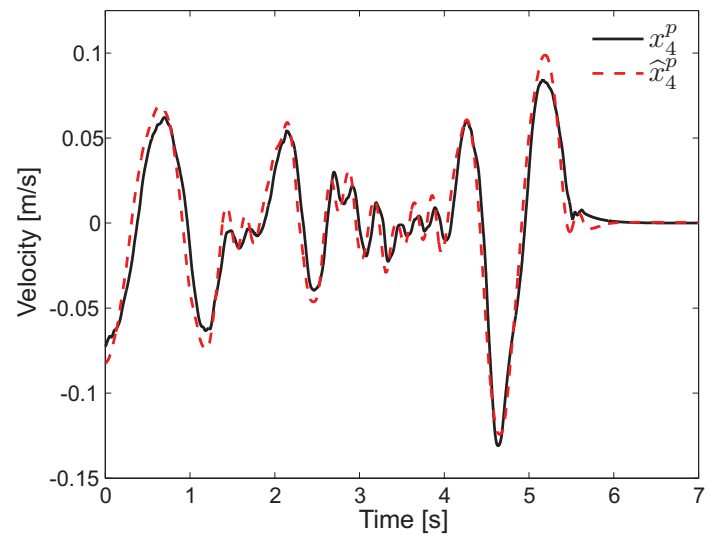

(d) Estimated versus actual velocity of the 2nd mass of subsystem $\mathbf{P}$

Fig. 6. State estimation experimental results of the state variables of subsystem/plant $\mathbf{P}$. The black line represents the measured states, whereas the red dashed lines represent the estimated states by the effort-based state observer. The observer depends on the availability of the state variables (velocity) and applied input of the subsystem A (Actuator) while keeping subsystem $\mathbf{P}$ free from any measurement (measurements are only taken to demonstrate the validity of the observer). Injecting the natural feedback effort-force onto the energy-based state observer structure allows for enforcing stable estimation error dynamics even in the absence of the state variable of the subsystem $\mathbf{P}$. Subsystem $\mathbf{A}$ is controlled to induce an arbitrary excitation into subsystem $\mathbf{P}$ to excite its state variables. The attached optical encoder to the system and the energy-based state observer provide measurements and estimations of the states of the first and second masses of subsystem $\mathbf{P}$, respectively.

ducted on a flexible dynamical system with four degreesof-freedom. The dynamical system shown in Fig. 1 and Fig. 5 represent the essence of many important dynamical systems, as complex nonlinear systems can be modeled by decomposing them into simpler subsystems that, upon interconnection add up their energies to determine the full behavior of the system. As shown in Fig. 5, the subsystem A with accessible state variables is considered to be a linear DC servomotor (FAULHABER, LM 1247 06001, Germany). This subsystem is connected via an elastic energy storage element with a flexible system with three degrees-of-freedom. Each degree-of-freedom is mounted on an almost frictionless linear slide (SCHNEEBERGER, ND 1-55.18, Germany). The state variables of this subsystem are conceptually considered inaccessible for measurement. In other words, we attempt to estimate its dynamical state variables without measurements. However, precise optical encoders are attached to each degree-of-freedom to compare the estimated states with the actual measurements. Each degree-of-freedom is equipped with an optical incremental linear encoder (RENISHAW, RGH26R05N00A, United Kingdom) with a resolution of $0.5 \mu \mathrm{m}$. The linear DC servomotor (subsystem A) is controlled via a dSPACE controller board (dSPACE, DS1103 PPC Controller Board, Germany) to excite the states of the dynamical system during the estimation experiment. A single measurement is only taken from the Linear DC servomotor, i.e., velocity (position is measured and velocity is calculated through a first order lowpass filter with cut-off frequency of $600 \mathrm{rad} / \mathrm{s}$ ), which is used along with the applied input to calculate the natural feedback effort-force of subsystem $\mathbf{P}$ by the effort-force observer (5). The estimated natural feedback effort-force is then injected onto the energy-based state observer (9) to provide estimates of the dynamical state variables of subsystem P. Fig. 6 illus- 
trates the results of the state variables estimation experiment by the energy-based state observer (9). Figs. 6(a) and (c) show the estimated position and velocity of the first degreeof-freedom of subsystem $\mathbf{P}$ versus their measured values, whereas Figs. 6(b) and (d) illustrate the position and velocity of the second degree-of-freedom of subsystem $\mathbf{P}$ versus their measured values. Experimentally, the effort-force observer is designed to meet two criterions, namely fast convergence of the estimated states and attenuation of the measurement noise of the sensor attached to the subsystem A. Cut-off frequency of, $g_{e f f}=628 \mathrm{rad} / \mathrm{s}$ almost allows for achieving the aforementioned criterions. The gain vector of the energybased state observer utilized throughout our experimental work is $\left[\begin{array}{lllll}17.96 & 2.01 & 12.45-0.08 & 0.84-0.15-10.38 & 3.65\end{array}\right]$.

As shown in Fig. 6, the state estimation results of the first four state variables (the position and velocity of the first and second masses of subsystem $\mathbf{P}$ ) are indeed stable. These results indicate that even in the absence of state variables and outputs of a subsystem, a natural feedback can be estimated upon the power-conserving interconnection with another subsystem with accessible state variables. The natural feedback can be utilized in designing an energy-based state observer by injecting the natural feedback onto its structure to enforce stable asymptotic estimation error dynamics.

It is worth noting that the energy-based state observer consists of two observers in cascade, i.e., (5) and (9). Each induces certain amount of phase lag into the system. Therefore, the phase margin of this observer is crucial. Fig. 4 indicates that the phase margins of the observer based system are satisfactory, however this issue has to be considered during the design of the energy-based state observer through the design of the effort-force observer (5) and the selection of the gain vector of (9).

\section{CONCLUSIONS AND FUTURE WORK}

This work addresses the state estimation problem of the state variables of dynamical subsystems with inaccessible state variables. The power-conserving interconnections between these subsystems and other subsystems with accessible state variables allows for providing natural feedback in terms of flow or effort variables. This natural feedback can be considered as an alternative to the inaccessible state variables and can be further used in the design of the energybased state observer. The energy-based state estimation formalism allows for categorizing state observers into effortbased and flow-based state observers. Availability of either the effort or the flow variables allows for realizing stable state estimation for a class of dynamical subsystems with inaccessible/unknown state variables.

Stability margins of the proposed energy-based state observer are studied to show the tolerance with the parameter deviation and the induced phase lag. Convergence stability of the state estimation is analyzed and investigated experimentally using a frictionless lumped mass-spring system with four degrees-of-freedom.

Future work in this field should be extended to investigate the design of energy-based state observer for systems with non-linear dynamics. While the analysis and experiments presented in this work consider systems with linear dynamics, the energy-based state estimation formalism could be used to estimate the state variables of systems with non-linear dynamics. In addition, the energy-based state estimation formalism will be utilized in designing motion control systems for applications in which utilization of sensors is impractical.

\section{REFERENCES}

[1] F. Esfandiari and H. K. Khalil, "Output feedback stablilization of fully linearizable systems," International Journal of Control, vol. 56, no. 5 , pp. 1007-1037, 1992.

[2] E. Bullinger and F. Allgower, "An adaptive high-gain observer for nonlinear systems," in Proceedings of the International Conference on Decision and Control (CDC), Vol. 5, pp. 4348-4353, San Diego, USA, December 1997.

[3] J. H. Ahrens and H. K. Khalil, "High-gain observers in the presence of measurement noise: a switched-gain approach," Automatica, vol. 45, no. 4, pp. 936-943, April 2009.

[4] D. Luenberger, "An introduction to observers," IEEE Transactions on Automatic Control, vol. 16, no. 6, pp. 596-602, December 1971.

[5] V. I. Utkin, J. Guldner, and J. Shi, "Sliding mode control in electromechanical systems,", Taylor-Fracis, 1999.

[6] M. Darouch, "Existence and design of functional observers for linear systems," IEEE Transactions on Automatic Control, vol. 45, no. 5, pp. 940-943, May 2000.

[7] A. E. Rundell, S. V. Drakunov, and R. DeCarlo "A sliding mode observer and controller for stabilization of rotational motion of a vertical shaft magnetic bearing," IEEE Transactions on Control Systems Technology, vol. 4, no. 5, pp. 598-514, September 1996.

[8] S. E. Talole, J. P. Kolhe, and S. B. Phadke, "Extended-state-observerbased control of flexible-joint system with experimental validation," IEEE Transactions on Industrial Electronics, vol. 57, no. 4, pp. 14111419, April 2010.

[9] T. L. Fernando, H. M. Trinh, and L. Jennings, "Functional observability and the design of minimum order linear functional observers," IEEE Transactions on Automatic Control, vol. 55, no. 5, pp. 12681273, May 2010.

[10] M. Savia and H. N. Koivo, "Contact micromanipulation-survey of strategies," IEEE/ASME Transactions on Mechatronics, vol. 14, no. 4, pp. 504-514, August 2009.

[11] S.-K. Hung, E.-T. Hwu, M.-Y. Chen, and L.-C. FU, "Dual-stage piezoelectric nano-positioner utilizing a range-extended optical fiber fabry-perot interferometer," IEEE/ASME Transactions on Mechatronics, vol. 12, no. 3, pp. 291-298, June 2007.

[12] M. Rakotondrabe, Y. haddab, and P. Lutz, "Development, modeling, and control of a micro-/nanopositioning 2-Dof stick-slip device," IEEE/ASME Transactions on Mechatronics, vol. 14, no. 6, pp. 733745, December 2009.

[13] W. J. O'Connor and D. Lang, "Position control of flexible robot arms using mechanical waves," Journal of Dynamics Systems, Measurement, and Control, vol. 120, no. 3, pp. 334-339, 1998.

[14] W. J. O'Connor, "Wave-based analysis and control of lump-modeled flexible robots," IEEE Transactions on Robotics, vol. 23, no. 2, pp. 342-352, April 2007.

[15] A. Macchelli and C. Melchiorri, "Control by interaction of the timoshenko beam," in Proceedings of the IFAC Workshop on Lagrangian and Hamiltonian Methods for Nonlinear Control, 1997.

[16] R. Ortega, A. J. van der Schaft, I. Mareels, and B. Maschke, "Putting energy back in control," IEEE Control Systems Magazine, vol. 21, no. 2, pp. 18-33, April 2001.

[17] A. Macchelli, S. Stramigioli, A. J. van der Schaft, and C. Melchiorri, "Considerations on the zero-dynamics of port hamiltonian systems and application to passive implementation of sliding-mode control," in Proceedings of the IFAC World Congress on Automatic Control, Barcelona, Spain, 2002.

[18] S. Komada, N. Machii, and T. Hori, "Control of redundant manipulators considering order of disturbance observer," IEEE Transaction on Industrial Electronics, vol. 47, no. 2, pp. 413-420, April 2000.

[19] S. Katsura, Y. Matsumoto, and K. Ohnishi, "Modeling of force sensing and validation of disturbance observer for force control," IEEE Transactions on Industrial Electronics, vol. 54, no. 1, pp. 530-538, February 2007. 